\title{
Massive Young Clusters in Nearby Galaxies
}

\author{
Jesús Maíz-Apellániz \\ Nolan R. Walborn \\ Space Telescope Science Institute, 3700 San Martin Drive, Baltimore, \\ $M D$ 21218, USA
}

\begin{abstract}
We study the size distribution of massive young clusters (MYCs) and of the cavities around them. We discuss the existence of two cluster populations and we find evidences for molecular cloud confinement in the early stages of development.
\end{abstract}

\section{Description}

We have searched the HST archive for nearby MYCs outside the Local Group with at least one nebular and three continuum WFPC2 exposures. Fourteen clusters with substantial nebular emission in their surroundings have been found and three more with no warm ionized gas associated have been located within $5^{\prime \prime}$ of the members of the original sample. We have obtained the half-light radius and magnitude of each cluster. A morphological study of the associated warm ionized gas has also been carried out and the radii of the existing cavities have been measured.
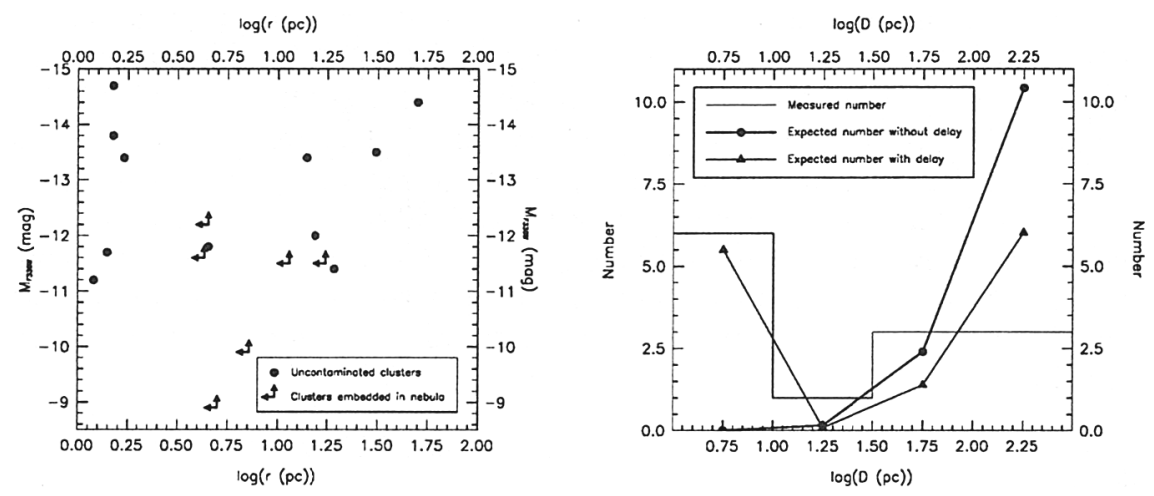

Figure 1. (left) WFPC2 U absolute magnitude vs. half-light radius plot for the clusters in the sample. The arrows indicate upper magnitude and radius limits for the clusters with filled nebular emission. (right) Cavity size histogram and expected number for wind-blown bubbles without time delay (no molecular cloud) and with a 2 Myr delay. 


\section{Results}

Table 1. Sample

\begin{tabular}{|c|c|c|c|c|c|c|c|}
\hline $\begin{array}{c}\text { Cluster } \\
\text { name }\end{array}$ & \multicolumn{2}{|c|}{$\begin{array}{c}\text { Cluster radius } \\
\left({ }^{\prime \prime}\right) \quad(p c)\end{array}$} & $\begin{array}{c}m_{\mathrm{F} 336 \mathrm{~W}} \\
(\mathrm{mag})\end{array}$ & $\begin{array}{c}M_{\mathrm{F} 336 \mathrm{~W}} \\
(\mathrm{mag})\end{array}$ & $\begin{array}{c}\text { Nebular } \\
\text { morphology }\end{array}$ & \multicolumn{2}{|c|}{ Cavity diameter } \\
\hline I Zw 18-I & 1.03 & 50.0 & 16.0 & -14.4 & shell & $0.3 \times 0.3$ & $190 \times 145$ \\
\hline I Zw 18-II & 0.40 & 19.2 & 19.0 & -11.4 & filled/shell & $0.3 \times 0.3$ & $39 \times 34$ \\
\hline UGC 5456-I-A & $0.55^{a}$ & 7.2 & 17.3 & -9.9 & filled & $<0.2$ & $<3$ \\
\hline UGC 5456-I-B & $0.38^{a}$ & 5.0 & 18.3 & -8.9 & filled & $<0.2$ & $<3$ \\
\hline NGC 1569-A1 & 0.14 & 1.5 & 14.8 & -14.7 & low emission & & \\
\hline NGC 1569-A2 & 0.16 & 1.7 & 16.2 & -13.4 & & & \\
\hline NGC 1569-C1 & 0.13 & 1.4 & 17.9 & -11.7 & shell & $\sim 2.7$ & $\sim 29$ \\
\hline NGC $1569-C 2$ & 0.11 & 1.2 & 18.4 & -11.2 & & & \\
\hline NGC 2363-A & $0.27^{a}$ & 4.5 & 17.4 & -12.2 & filled & $<0.5$ & $<8$ \\
\hline NGC 2363-B & 0.27 & 4.5 & 17.8 & -11.8 & shell & $3.9 \times 3.2$ & $64 \times 53$ \\
\hline NGC 4214-I-A & $0.07^{b}$ & 1.5 & 14.5 & -13.8 & shell & $9.7 \times 8.0$ & $194 \times 160$ \\
\hline NGC 4214-I-B & 1.56 & 31.0 & 14.8 & -13.5 & shell & $8.5 \times 3.0$ & $170 \times 60$ \\
\hline NGC 4214-II-A & $0.87^{a}$ & 17.4 & 16.8 & -11.5 & filled & $<0.5$ & $<10$ \\
\hline NGC 4214-II-B & $0.57^{a}$ & 11.4 & 16.8 & -11.5 & filled & $<0.1$ & $<2$ \\
\hline NGC 4214-II-C & 0.78 & 15.4 & 16.3 & -12.0 & filled/shell & $2.0 \times 2.0$ & $40 \times 40$ \\
\hline NGC 5253-IV & 0.70 & 13.9 & 15.0 & -13.4 & low emission & - & - - \\
\hline NGC 5253-V & $0.21^{a}$ & 4.3 & 16.8 & -11.6 & filled & $0.3 \times 0.3$ & $6 \times 6$ \\
\hline
\end{tabular}

$a$ Radii and magnitudes are only upper limits due to differential extinction and contamination of the continuum filters by nebular continuum and line emission.

$b$ Marginally resolved.

The clusters without filled nebular emission seem to follow a bimodal distribution centered around $\log r \sim 0.15(r \sim 1.4 \mathrm{pc})$ and $\log r \sim 1.3(r \sim 20 \mathrm{pc})$ with only one lower luminosity case (NGC 2363-B) in the intermediate region. These two cluster types correspond to Super Star Clusters (SSCs) and Scaled OB Associations (SOBAs). No similar trend is visible in the more uncertain measurements of the nebulosity-filled clusters.

The compact clusters include three previously identified SSCs (NGC 1569A1 and A2, and NGC 4214-I-A) and two lower luminosity objects (NGC 1569$\mathrm{C} 1$ and $\mathrm{C} 2$ ). An extended halo (with a SOBA-like structure) surrounds those objects and is especially prominent in the case of NGC 4214-I-A. The radii and magnitudes listed are those of the cluster cores.

The most relevant massive young clusters in the Local Group can be fit into the bimodal pattern, with R136 and NGC 3603 belonging to the compact class and NGC 604 and NGC 595 to the extended one. The R136 halo (the 30 Doradus cluster) is even more prominent than that of NGC 4214-I-A while that of NGC 3603 is very weak.

The distribution of bubble sizes does not agree with the expectations of wind-blown bubble models with no external pressure and a uniform age distribution. The number of filled and filled/shell (i.e. younger) objects is higher than expected relative to shell (i.e. older) ones in a way which is incompatible with a selection effect explanation. The agreement is much better if a $2 \mathrm{Myr}$ old delay is included to account for molecular cloud confinement.

Go to http://www.stsci.edu/ jmaiz/manchester.html to obtain the color figures in this poster. 